\title{
Hallazgos y Propuesta de Sistematización de las Variaciones Quirúrgicamente Importantes de la Arteria Cística en un Estudio in vivo en 2000 Colecistectomías Laparoscópicas Ambulatorias
}

\author{
Findings and Proposal for Systematization of Surgically Important Variations of the Cystic \\ Artery Based on an in vivo Study of 2000 Outpatient Laparoscopic Cholecystectomies
}

\author{
Noguera Miguel Angel ${ }^{1,2}$; Romero Cesar Alejandro ${ }^{1}$; Martinez Aldo Gustavo ${ }^{1}$; \\ Diaz San Roman Hugo ${ }^{1}$; Rotger Mariano ${ }^{1}$ \& Espeche Federico ${ }^{1}$
}

NOGUERA, M. A.; ROMERO, C. A.; MARTINEZ, A. G.; DIAZ, S. R. H.; ROTGER, M. \& ESPECHE, F. Hallazgos y propuesta de sistematización de las variaciones quirúrgicamente importantes de la arteria cística en un estudio in vivo en 2000 colecistectomías laparoscópicas ambulatorias. Int. J. Morphol., 38(1):30-34, 2020.

RESUMEN:Realizar un estudio anatómico in vivo con la especial y pequeña disección quirúrgica durante una colecistectomía laparoscópica sobre las variaciones de la arteria cística. Estudio prospectivo de 38 meses, en 2000 pacientes consecutivos sometidos a colecistectomía laparoscópica programada, sin signos de inflamación aguda, ni alteración que impida disección y correcta evaluación del triángulo hepatocístico. Se disecó quirúrgicamente identificándose la arteria cística y posible duplicación, eran clínicamente importantes aquellas con diámetro mayor a 1,5 mm, requerían maniobra hemostática. Se anotaron los hallazgos en planilla especial a los fines del presente estudio. En 1831 casos había arteria única en medio del triángulo hepatocístico. Hubo 169 variaciones $(8,45$ \%). En 97 casos: doble vascularización, con una arteria en situación normal y otra ubicada lateralmente al triangulo hepatocístico. En 44 pacientes había una arteria única lateralmente al conducto cístico que no lo cruzaba nunca. En 22 casos existía una arteria cruzando el colédoco y el cístico entrando en el triángulo. En 6 oportunidades una doble arteria, una en el triángulo hepatocístico y otra lateralmente que no cruzaba el cístico ni colédoco. En una oportunidad se observó una sola arteria importante que salía directamente de la placa cística entre segmento 4 y 5 , y en otro caso solo pequeñas arterias proveniente de la placa cística. Podemos dividirlas en arterias únicas o dobles, en base exclusiva a la necesidad de maniobra hemostática. Podemos decir que las variaciones estarán presentes en aproximadamente 1/12 casos y necesitará una maniobra hemostática especial en 1/20 casos

PALABRAS CLAVE: Arteria cística; Variaciones anatómicas; Colecistectomía; Triángulo hepatocístico.

\section{INTRODUCCIÓN}

La colecistectomía laparoscópica es por lejos la cirugía más común de cualquier servicio de cirugía general, desde hace años solo se realiza en forma laparoscópica en nuestro medio, lo que permite una mejor visualización de los elementos anatómicos involucrados debido a la magnificación y la resolución actual de los sistemas de video laparoscopía.

Esta cirugía requiere una atención cuidadosa y delicada, con especial atención en la posibilidad de encontrar variaciones anatómicas o anomalías en las vías biliares y las estructuras vasculares involucradas en la cirugía. La mala interpretación de la anatomía percibida más que una falta en la destreza quirúrgica es la causa más asociada con lesiones de la vía biliar durante la colecistectomía (Algieri et al., 2014).
Si bien se propusieron numerosas estrategias para minimizar los riesgos de lesión quirúrgica, la más popularizada es la llamada "visión crítica" propuesta por Strasberg et al. (1995).

Existen numerosos estudios anatómicos sobre la irrigación de la vesícula biliar, pero para su realización exigen una disección mucho más grande que la realizada quirúrgicamente durante una colecistectomía laparoscópica, en la cual la disección de las estructuras peritoneales hepatocísticas y su contenido es muy limitada. Eso supone que muchas de las variaciones no son correctamente visualizadas, como por ejemplo un origen alejado o anómalo de la arteria cística, por ejemplo, originándose en la

\footnotetext{
${ }^{1}$ Hospital de Día Pte. Néstor Kirchner. Mendoza 128. San Miguel de Tucumán, Tucumán, Argentina.

${ }^{2}$ Cátedra de Anatomía Normal. Facultad de Medicina. Universidad Nacional de Tucumán, Tucumán, Argentina.
} 
NOGUERA, M. A.; ROMERO, C. A.; MARTINEZ, A. G.; DIAZ, S. R. H.; ROTGER, M. \& ESPECHE, F. Hallazgos y propuesta de sistematización de las variaciones quirúrgicamente importantes de la arteria cística en un estudio in vivo en 2000 colecistectomías laparoscópicas ambulatorias. Int. J. Morphol., 38(1):30-34, 2020.

gastroduodenal o la hepática propia. Dicha variación asimismo tiene interés anatómico, pero no quirúrgico, ya que si su ingreso a la vesícula es a través del triángulo de BuddeCalot y su terminación normal, pasa desapercibido en la disección quirúrgica, y carece prácticamente de interés para la realización de la colecistectomía.

El objetivo del presente trabajo fue estudiar prospectivamente la arteria cística dentro del trígono hepatocístico e identificar las variaciones anatómicas de este vaso que se pueden encontrar durante los particulares límites de la disección quirúrgica laparoscópica y que tengan un neto interés quirúrgico, proponiéndola sistematizarlas de una forma más sencilla y práctica.

\section{MATERIAL Y MÉTODO}

Se realizó un estudio prospectivo, descriptivo, en un periodo de 38 meses en el Servicio de Cirugía General del Hospital de Dia Pte. Néstor Kirchner de Tucumán, Argentina, en pacientes que eran sometidos a colecistectomía laparoscópica ambulatoria programada. Todos los pacientes prestaron su conformidad para la obtención de datos estadísticos intraoperatorios anónimos y/o registros gráficos de acuerdo a los lineamientos del Código de Ética de la Asociación Médica Mundial (Declaración de Helsinski) a los efectos del presente y otros trabajos que no significaban experimentación alguna ni cambio de conducta terapéutica y avalado por el comité de docencia e investigación de la institución.

Se consideraron candidatos a todos los pacientes que serían sometidos a colecistectomía laparoscópica ambulatoria programada por litiasis vesicular. Se incluyeron todos los pacientes que no presentaban signos de inflamación aguda o adherencias que pudieran alterar la evaluación del trígono hepatocístico y la arteria cística, lo que constituye condiciones clínicas que están comprendidos en el tipo A de la clasificación de Torres et al. (1992) propuesta por la Asociación Argentina de Cirugía. Se excluyeron a 534 pacientes por la presencia de inflamación y/o alteración anatómica adquirida, quedando comprendidos 2000 pacientes, que era el punto de corte propuesto al comenzar el estudio.

En todos los casos se procedió a la disección quirúrgica minuciosa del trígono hepatocístico, comenzando por su cara posterior generalmente, luego por su cara anterior, llegando la disección cercana a la vesícula biliar, hasta la visualización correcta de la visión crítica de seguridad (Strassberg, 1995), siempre procurando visualizar adecuadamente conducto cístico, ganglio cístico y arteria cística, anotando su posición, número y localización. Se consideró como clínicamente importantes las arterias con diámetro mayor a $1,5 \mathrm{~mm}$ (más grande que la punta del electrocauterio laparoscópico o Hook), y que requirieron algún tipo de maniobra hemostática (clipado y/o electrocoagulación), la disección era exclusivamente quirúrgica, no permitiéndose la disección por fuera de los límites del triángulo hepatocístico para evitar lesiones.

A todos los pacientes luego de la realización de la cirugía y la confección de su protocolo quirúrgico informatizado, se recogieron sus datos en planilla adjunta manual normatizada a los fines exclusivos del presente trabajo, donde constaban sus datos clínicos, evaluación preoperatoria, hallazgos intraoperatorios, en especial las variaciones de la arteria cística en forma gráfica (Fig. 1), usando como guía la clasificación propuesta por Smadja \& Blumgart (1988), de referencia común en cirugía hepatobiliar, en el formulario se debía constar el tipo de arteria y contenía la posibilidad de anotar alguna no comprendida en dichos grupos.

El análisis de los datos se pasó para su estudio a una planilla de Excel con doble control de entrada de datos. Obteniéndose los resultados que se mostrarán a continuación.

\section{RESULTADOS}

Se incluyeron un total de 2000 pacientes en los cuales no existían alteraciones inflamatorias evidentes del triángulo hepatocístico, y que se pudo realizar una correcta visualización de la arteria cística y estructuras vecinas.

Siguiendo la clasificación propuesta por Smadja \& Blumgart, se encontró una configuración de la arteria cística que se considera normal, es decir la arteria ubicada en medio del triángulo hepatocístico conformando el límite superior del triángulo de Calot (Fig. 1-A) en 1831 casos (91,55 $\%)$. Su origen supuesto es en la arteria hepática derecha, lo cual no puede ser observado en la disección quirúrgica que se realiza normalmente durante una colecistectomía. Eso puede causar la observación intraoperatoria de que no observamos ninguna arteria originada de la hepática izquierda (Fig. 1-E), ocasionado probablemente por el déficit de la disección quirúrgica en sentido interno, pero esto no conlleva cambio en las maniobras hemostáticas intraoperatorias.

La presencia de una arteria ubicada lateralmente al conducto cístico por fuera del triángulo hepatocístico como se observa en la Figura 1-B, pero seguida de otra arteria 
ubicada dentro del trígono en posición normal, es decir el hallazgo de una doble vascularización, fue observada en 96 casos $(4,8 \%)$, siendo la más frecuente de las variaciones observadas. Esta disposición plantea al cirujano la necesidad de una doble maniobra hemostática (coagulación y/o clampeo), lo cual es importante durante una cirugía.

Otra configuración encontrada en 44 casos $(2,2 \%)$ fue la presencia de una arteria ubicada lateralmente al con- ducto cístico que se dirigía oblicuamente hacia abajo y adentro y que nunca cruzaba el cístico (Fig. 1-C) estando siempre por fuera del triángulo hepatocístico. Deducimos según el esquema de Smadja \& Blumgart que podría originarse de la arteria hepática común, lo cual no se puede ver durante la disección quirúrgica sin aumentar los riesgos de daño innecesariamente. No se observó una segunda arteria dentro del trígono hepatocístico, lo cual lo diferencia de la variante precedente (Fig. 1-B).

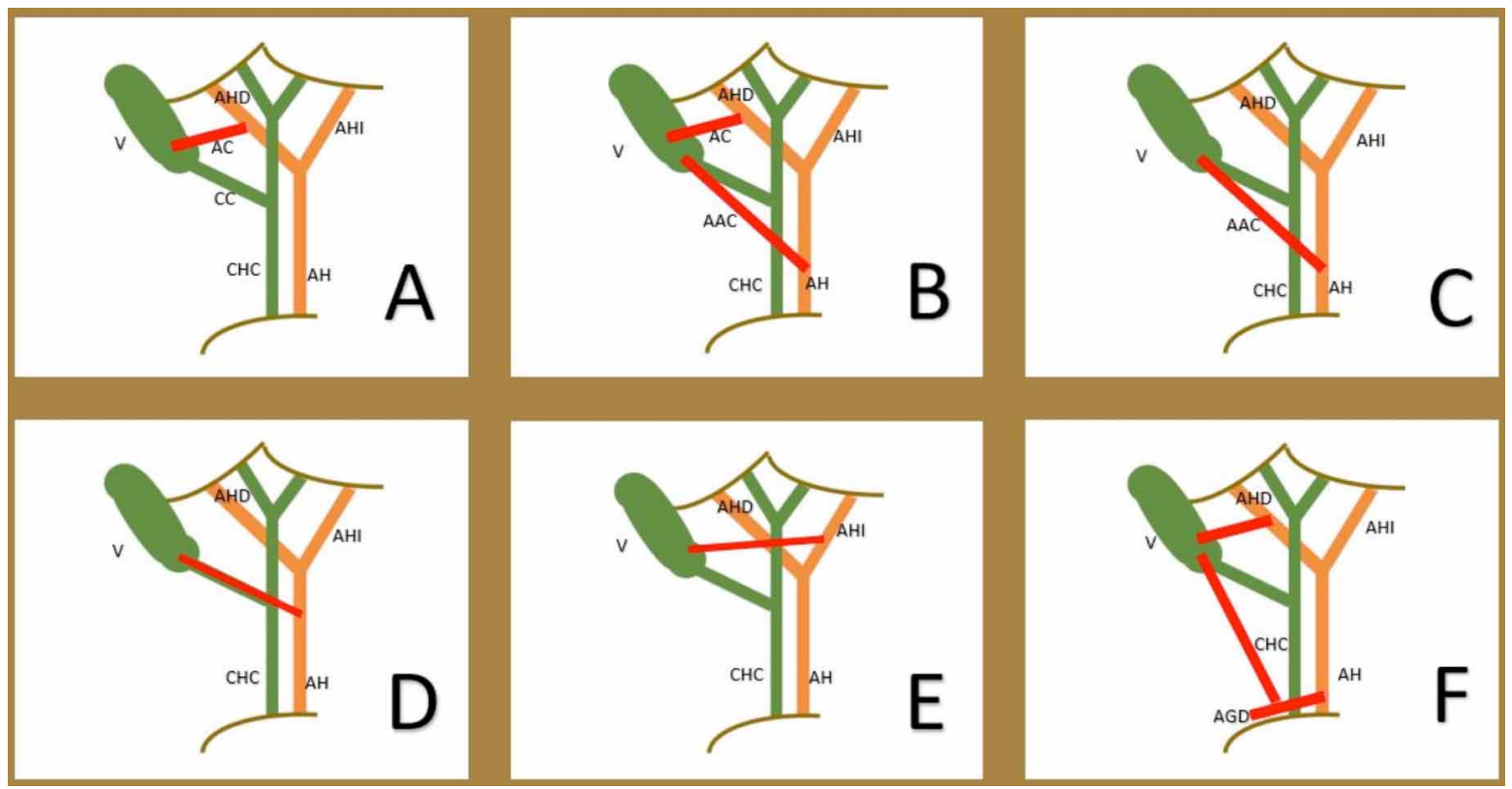

Fig. 1. Esquema de las variaciones comunes de la arteria cística.Referencias: V: Vesícula biliar, AHD: Arteria hepática derecha, AHI: Arteria hepática izquierda, AC: Arteria Cística, AAC: Arteria Accesoria Cistica, AGD: Arteria Gastroduodenal, CHC: Conducto Hepatocolédoco. Variaciones A-F descripción detallada en el texto.

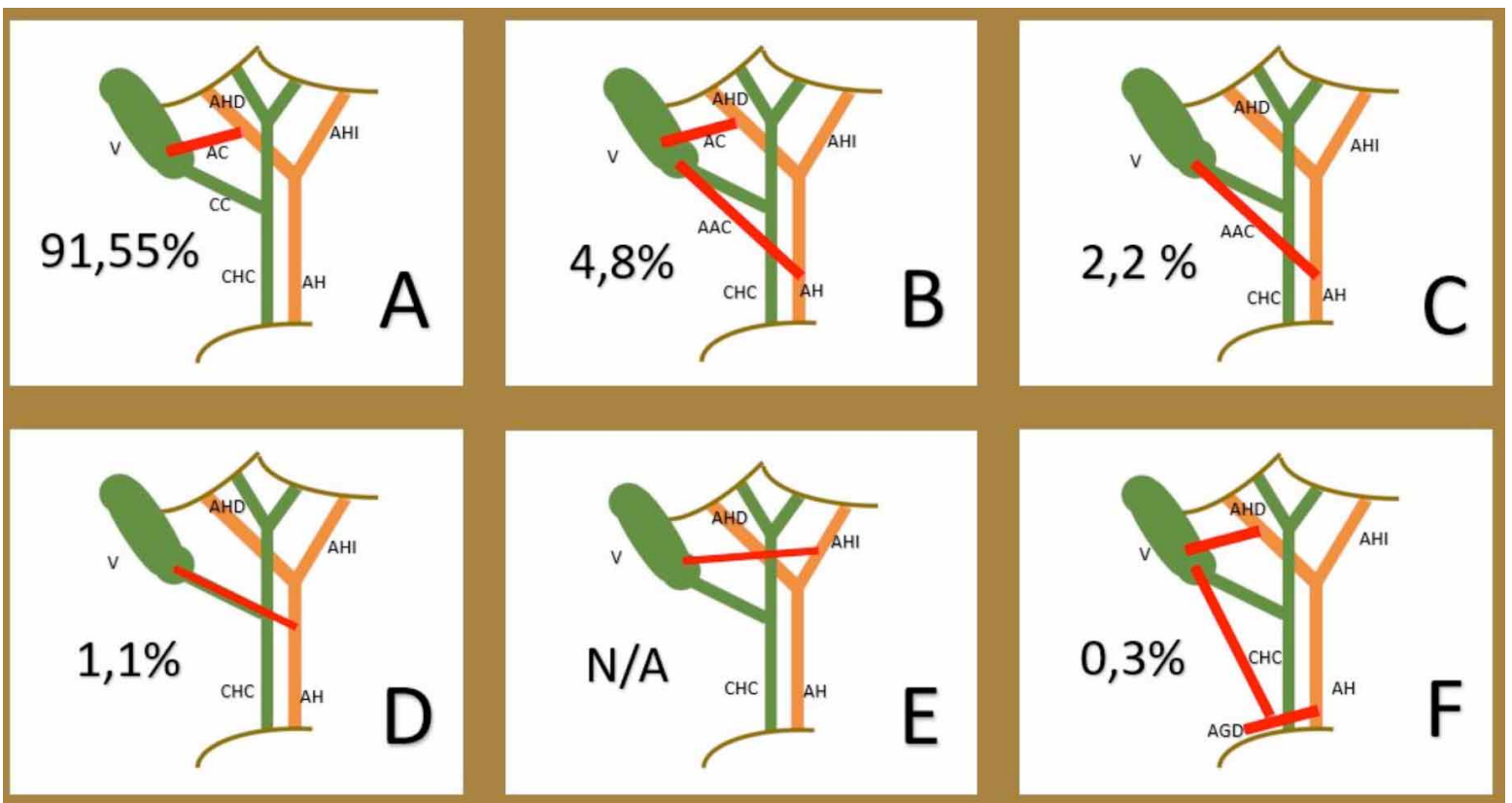

Fig. 2. Variaciones encontradas, expresadas en porcentaje del total sobre 2000 casos. Posee las mismas referencias que la Fig. 1. 
La presencia de una arteria cística ubicada dentro del triángulo hepatocistico, cerca de la desembocadura, cruzando la cara anterior del colédoco para dirigirse transversalmente por encima de la desembocadura del cístico (Fig. 1-D) se encontró en 22 oportunidades (1,1\%). Se desprende que su origen más probable es la arteria hepática común.

En 6 oportunidades $(0,3 \%)$ se pudo observar una doble arteria, una en el límite superior del triángulo hepatocistico pegada al margen hepático y otra externamente al conducto cístico que se dirige hacia abajo y adentro, cruzando la vía biliar principal por debajo de la desembocadura del cístico o llegando al ángulo duodenal superior (Fig. 1-F). Nuestro esquema de referencia deduce que su origen es la arteria gastroduodenal, pero eso no es explorado durante la disección quirúrgica.

Hubo dos casos de variación cística $(0,1 \%)$ que no pudo ser categorizado en ninguno de los esquemas propuestos, en el primer caso se observó una arteria que recorría el margen superior del triángulo sobre el margen inferior del hígado, y en el segundo no se observaba ninguna arteria cística importante dentro o fuera del triángulo hepatocistico, sino que había pequeños ramos que recorrían la cubierta peritoneal vesicular, proveniendo directamente de la placa cística.

\section{DISCUSIÓN}

Los resultados encontrados nos permiten decir que se observarán variaciones a la arteria cística en un 8,45\% de los casos durante una disección quirúrgica laparoscópica clásica (Fig. 2). El número encontrado explica las particularidades que se encuentran en una disección quirúrgica limitada a la parte más lateral del trígono hepatocístico, con lo cual todas las variaciones que se encuentran en las disecciones anatómicas cadavéricas quedan descartadas al no poder observarlas (por ejemplo, en los orígenes y trayectos iniciales de la arteria cística).

Tomando en cuenta los hallazgos observados en estos 2000 casos podemos establecer una forma de aproximación quirúrgica a estas variaciones, poniendo énfasis en las maniobras hemostáticas y no en la mera observación anatómica básica, por lo que encontramos una diferencia importante de un estudio básico anatómico y otro clínico in vivo.

Se observa que, de las 169 variaciones observadas, 96 de ellas $(4,8 \%)$ requerirán una maniobra hemostática doble al existir más de una arteria cística (Tipo B+F de la Figura 1), independientemente del origen de la misma.
En las restantes 73 variaciones la arteria es simple y su maniobra hemostática única es muy similar entre ellas (Tipo C+D+E de la Figura 1), el cual también es casi idéntico a cuando la arteria se encuentra en su origen y posición habitual.

Cuando la arteria se encuentra por fuera del triángulo hepatocístico, externamente al conducto cístico (tipos BC-D-F) hay que tener presente que en un $56,8 \%$ de las veces $(96 / 169)$ se encuentra una segunda arteria que necesitará alguna maniobra hemostática.

En este punto es que con criterio netamente quirúrgico podemos decir que las variaciones de la arteria cística solo las hemos encontrado en un 8,45\% del total de los pacientes, ellas se pueden dividir en arterias Únicas (Tipo $\mathrm{C}+\mathrm{D}+\mathrm{E}+\mathrm{Otras}$ ) que corresponden al 56,8 \% de las mismas (96/169), y las arterias Dobles (Tipo B+F) que corresponden al 43,2\% de los casos (73/169), es decir que la presencia de una variación es de aproximadamente 1 de cada 12 casos, pero lo más importante es que solo 1 de cada 20 casos necesitará una maniobra hemostática doble o especial.

Consideramos a este punto de vista más práctico para el cirujano general que aborde la realización de la colecistectomía. Las múltiples variaciones descriptas en la literatura anatómica (Balija et al., 2001; Mlakar et al., 2003; Ding et al., 2007) no siempre tienen su importancia durante la realización de la cirugía, ni pueden ser correctamente evaluadas con la pequeña disección que se lleva a cabo durante el acto quirúrgico.

NOGUERA, M. A.; ROMERO, C. A.; MARTINEZ, A. G.; DIAZ, S. R. H.; ROTGER, M. \& ESPECHE, F. Findings and Proposal for systematization of surgically important variations of the cystic artery based on an in vivo study of 2000 outpatient laparoscopic cholecystectomies. Int. J. Morphol., 38(1):30-34, 2020.

SUMMARY: This is an anatomical study with the special and small dissection of a laparoscopic cholecystectomy on the surgically important variations of the cystic artery. A prospective, 19 -month study was conducted in 2000 , including consecutive patients undergoing programmed laparoscopic cholecystectomy, without signs of acute inflammation, or alteration, that would prevent dissection and correct evaluation of the cystohepatic triangle. It was surgically dissected, identifying the main cystic artery and its possible collateral arteries. Those with a diameter greater than $1.5 \mathrm{~mm}$ being considered as clinically important, requiring haemostatic maneuver (clipping and / or electrocoagulation). The findings were recorded on a special form for the purposes of this study. The classic, single-artery arrangement in the middle of the cystohepatic triangle was found in 1831 cases. The variations found were $169(8.45 \%)$. In 97 cases there was 
double vascularization, with one artery in normal position and another outside the cystic duct. In 44 patients, a single artery that did not cross the cystic was observed. In 22 cases an artery outside the cystic but crossing it before the duct. In 6 cases a double artery, one in the cystohepatic triangle and another outside the triangle, did not cross the cystic or the bile duct. In one instance, a single major artery was seen emerging directly from the cystic plaque between segments four and five. These can be divided into single or double arteries, based exclusively on the need for hemostatic maneuver. Knowledge of anatomical variations of the cystic artery is important for the surgeon. The variation presents in 1 of 12 cases, and requires a special hemostatic maneuver in 1 of 20 cases

KEY WORDS: Cystic artery; Variations; Anatomical; Cholecystectomy; Hepatocystic Triangle.

\section{REFERENCIAS BIBLIOGRÁFICAS}

Algieri, R. D.; Ferrante, M. S.; Ugartemendía, J. S.; Bernadou, M. M.; Pina, L. \& Álvarez Escalante, A. Trigonum cystohepaticum: critical anatomical area for surgical safety. Int. J. Morphol., 32(3) :860-5, 2014.

Balija, M.; Huis, M.; Stulhofer, M. \& Nikolic, V. Contribution to the nomenclature of variations of the cystic artery. Chirurg., 72(2):154-8, 2001.

Ding, Y. M.; Wang, B.; Wang, W. X.; Wang, P. \& Yan, J. S. New classification of the anatomic variations of cystic artery during laparoscopic cholecystectomy. World. J. Gastroenterol., 13(42):5629-34, 2007.

Mlakar, B.; Gadzijev, E. M.; Ravnik, D. \& Hribernik, M. Anatomical variations of the cystic artery. Eur. J. Morphol., 41(1):31-4, 2003.

Smadja, C. \& Blumgart, L. H. The Biliary Tract and the Anatomy of Biliary Exposure. En: Blumgart, L. H. (Ed.). Surgery of the Liver and Biliary Tract. Edinburgh, Churchill Livingstone, 1988.

Strasberg, S. M.; Hertl, M. \& Soper, N. J. An analysis of the problem of biliary injury during laparoscopic cholecystectomy. J. Am. Coll. Surg., 180(1):101-25, 1995.

Torres, R. A.; Beltrame, O. \& Orban, R. Propuesta de clasificación laparoscopica de la patologia vesicular y su correlación con los resultados quirúrgicos. Rev. Argent. Cir., 63(5):150-6, 1992.
Dirección para correspondencia

Dr. Miguel Angel Noguera

Mendoza $1281^{\circ}$ piso Oficina 3

Tucumán

ARGENTINA

Email: mnoguera@intramed.net

Recibido : 20-06-2019

Aceptado: 25-07-2019 